\title{
Existence and exponential stability in the $p$ th moment for impulsive neutral stochastic integro-differential equations driven by mixed fractional Brownian motion
}

\author{
Xia Zhou ${ }^{1 *}$, Dongpeng Zhou' and Shouming Zhong ${ }^{2}$
}

"Correspondence:

zhouxia44185@163.com

'School of Mathematics and

Computing Science, Guilin

University of Electronic Technology,

Guilin, China

Full list of author information is

available at the end of the article

\begin{abstract}
This paper consider the existence, uniqueness and exponential stability in the pth moment of mild solution for impulsive neutral stochastic integro-differential equations driven simultaneously by fractional Brownian motion and by standard Brownian motion. Based on semigroup theory, the sufficient conditions to ensure the existence and uniqueness of mild solutions are obtained in terms of fractional power of operators and Banach fixed point theorem. Moreover, the pth moment exponential stability conditions of the equation are obtained by means of an impulsive integral inequality. Finally, an example is presented to illustrate the effectiveness of the obtained results.
\end{abstract}

Keywords: Neutral stochastic differential equations; Impulsive; Exponential stability; Fractional Brownian motion; Wiener process

\section{Introduction}

The differential equation is an important tool to describe the law of development and change of things, and random disturbance is inevitable, thus many practical problems can be modeled by stochastic differential equations. The existence, uniqueness and stability of solutions of stochastic differential equations have been studied by many researchers [1-10]. Besides, many dynamical systems include not only time delays but also derivatives with delays. Neutral differential equations, firstly introduced by Hale and Meyer [11], are used to describe this kind of systems. It worth pointing out that in many cases the time delay or derivative with delay is the source of instability in dynamical systems. So, many researchers have increased their interests in investigating the stability of neutral stochastic functional differential equations (NSFDEs) with delays, for instance, Chen [12] discussed the exponential stability in $p$ th moment and almost surely exponential stability of mild solutions for NSFDEs. Boufoussi and Hajji [13] considered the existence and asymptotic behavior of mild solutions for NSFDEs in Hilbert space. Very recently, Zhang and Ruan [14] studied the existence, uniqueness and exponential stability in the $p$ th moment of mild solution for NSFDEs driven by mixed fractional Brownian motion. However, the impulsive effects were not considered in $[12,13]$ and [14].

(c) The Author(s) 2019. This article is distributed under the terms of the Creative Commons Attribution 4.0 International License (http://creativecommons.org/licenses/by/4.0/), which permits unrestricted use, distribution, and reproduction in any medium, provided you give appropriate credit to the original author(s) and the source, provide a link to the Creative Commons license, and indicate if changes were made. 
Impulsive phenomena have gained a lot of attention in the last decades due to their wide applications, Lakshmikantham [15] and Samoilenko [16] elaborated on the theory and application of impulsive differential equations in their monographs. In addition, the approximate controllability [17, 18], global attractiveness and quasi-invariant sets [19], existence and stability [20-25] of the impulsive stochastic differential equation were investigated. Furthermore, the concept "aftereffect" introduced in scientific and engineering disciplines is very important, it is well known that the integro-differential equation is an effective tool to model a system with aftereffect. Recently, Ma et al. [23] discussed the existence, uniqueness and mean-square exponential stability of a mild solution of impulsive neutral stochastic integro-differential equation. Ren et al. [26] proved the existence and uniqueness of mild solutions for impulsive neutral stochastic integro-differential equation under the assumptions of non-Lipschitz conditions.

As a Gaussian stochastic process, fractional Brownian motion heavily relies on the Hurst index $H \in(0,1)$ introduced by Kolmogorov [27], it is an effective tool in modelling many stochastic systems since its stationary increments and long-range dependence properties. Such as financial market time series displays long-range dependence and momentum. Long-range dependence has an important feature, which explains the well-documented evidence of volatility persistence, especially when the Hurst index $H \in(1 / 2,1)$, which is most frequently encountered in real financial data. Peters [28] pointed out the exchange rate of the dollar against the Japanese yen from January 1972 to December 1990 followed the fBm with Hurst index $H=0.642$. One can study the option pricing problem when the option contract is driven by a geometric $\mathrm{fBm}$. A fractional Brownian motion reduces to a standard Brownian motion when $H=1 / 2$. Due to the good nature and wide application of $\mathrm{fBm}$, the differential equations driven by $\mathrm{fBm}$ have been studied by many scholars and have been reported in the literature $[4,5,7,9,13,14,17-26]$. Mixed $\mathrm{fBm}$ is a stochastic process that contains both standard Brownian motion and fractional Brownian motion, which has gained a lot of attention since Cheridito [29] had studied the statistical properties of it. It is an effective tool in different applications. For instance, we can use mixed $\mathrm{fBm}$-driven stochastic differential equations to model systems that contain not only white random noise but also long memory random noise. In the field of finance, one can distinguish between microstructure and randomness coming from the economical situation. In recent years, there were many researchers who discussed the stochastic differential equations driven by mixed $\mathrm{fBm}$; see [14, 30-33] and the references therein.

In recent years the NSFDEs with impulsive effects have received much attention since they have wide applications, but the corresponding theory has not been fully explored. To the best of our knowledge, there is no paper considering the exponential stability of impulsive neutral stochastic integro-differential equation driven by mixed fractional Brownian motion. Thus we consider the following impulsive neutral stochastic integro-differential equation driven by mixed fractional Brownian motion:

$$
\left\{\begin{array}{l}
d\left[x(t)-p\left(t, x_{t}\right)\right] \\
\quad=\left[A x(t)+f\left(t, x_{t}\right)+\int_{0}^{t} h\left(t, s, x_{s}\right) d s\right] d t+g\left(t, x_{t}\right) d W(t) \\
\quad+\sigma(t) d B^{H}(t), \quad t \in[0,+\infty), t \neq t_{k}, \\
\Delta x\left(t_{k}\right)=I_{k}\left(x\left(t_{k}^{-}\right)\right), \quad t=t_{k}, k=1,2, \ldots, \\
x(t)=\phi(t) \in \mathcal{P C}([-r, 0] ; X), \quad t \in[-r, 0],
\end{array}\right.
$$


where $A$ is the infinitesimal generator of an analytic semigroup $(T(t))_{t \geq 0}$ of bounded linear operators in a Hilbert space $X, W(t)$ and $\left\{B^{H}(t), H \in\left(\frac{1}{2}, 1\right)\right\}$ are for standard Brownian motion (also called Wiener process) and fractional Brownian motion, respectively, in this paper, they are mutually independent. The impulsive moments $t_{k}$ satisfy $0<t_{1}<t_{2}<\cdots<t_{k}<$ $\cdots$, and $\lim _{k \rightarrow+\infty} t_{k}=\infty . I_{k}: X \rightarrow X, \Delta x\left(t_{k}\right)=x\left(t_{k}^{+}\right)-x\left(t_{k}^{-}\right)$represents the jump size in the state $x$ at $t_{k}$, where $x\left(t_{k}^{+}\right)$and $x\left(t_{k}^{-}\right)$are the right and left limits of $x(t)$ at $t_{k}$, respectively. The initial state $\phi \in \mathcal{P C}([-r, 0] ; X)$ (the space of all functions that are continuous everywhere except for a finite number of points from $[-r, 0]$ to $X)$ and $\|\phi\|_{\mathcal{P C}}=\sup _{s \in[-r, 0]}\|\phi(s)\|<+\infty$. For every continuous function $x(t), t \in[0,+\infty)$, we denote by $x_{t}$ the element of $\mathcal{P C}$ defined by $x_{t}(\theta)=x(t+\theta),-r \leq \theta \leq 0$. The functions $p:[0,+\infty) \times \mathcal{P C} \rightarrow X, f:[0,+\infty) \times \mathcal{P C} \rightarrow X$, $g:[0,+\infty) \times \mathcal{P C} \rightarrow X, h:[0,+\infty) \times[0,+\infty) \times \mathcal{P C} \rightarrow X$, and $\sigma:[0,+\infty) \rightarrow L_{B}^{0}(Y, X)$ are all Borel measurable, we will specify this later. Our purpose of this paper is to consider the existence, uniqueness and exponential stability in $p$ th moment of mild solution of system (1).

The rest of this paper is organized as follows. In Sect. 2, we recall some basic concepts and lemmas needed in this paper. In Sect. 3, we get the sufficient conditions that ensure the existence and uniqueness of mild solution of system (1). In Sect. 4, we discuss the exponential stability in $p$ th moment by an impulsive integral inequality. In Sect. 5, we give an example to illustrate the effectiveness of the obtained results. In Sect. 6, we present our conclusion.

\section{Preliminaries}

In this section, we introduce some notations and recall the basic concepts and lemmas as regards the Wiener process, fractional Brownian motion and the Wiener integral with respect to fractional Brownian motion. Furthermore, we state some basic properties of analytic semigroups and fractional powers of their infinitesimal generators.

Let $(\Omega, \mathcal{F}, \mathbb{P})$ be a complete probability space satisfying the normal assumptions, the mathematical expectation operator is denoted by $E(\cdot)$. Denote $W=\{W(t), t \in[0,+\infty)\}, B=$ $\left\{B^{H}(t), t \in[0,+\infty)\right\}$ by the standard Brownian motion and fractional Brownian motion with Hurst parameter $H \in(1 / 2,1)$, respectively. Let $X, K_{i}(i=W, B)$ denote separable Hilbert spaces, and let $L\left(K_{i}, X\right)$ be two spaces of all bounded linear operators from $K_{i}$ to $X$. We assume that $\left\{e_{n}^{(i)}\right\}_{n \in N^{+}}$are complete orthonormal bases in $K_{i}$. Let $Q^{(i)} \in L\left(K_{i}, K_{i}\right)$ be two non-negative self-adjoint operators defined by $Q^{(i)} e_{n}^{(i)}=\lambda_{n}^{(i)} e_{n}^{(i)}$ with finite trace $\operatorname{tr} Q^{(i)}=\sum_{n=1}^{\infty} \lambda_{n}^{(i)}<\infty$, where $\left\{\lambda_{n}^{(i)}\right\}_{n \in N^{+}}$are non-negative real numbers. Let $L_{i}^{0}\left(K_{i}, X\right)$ be the spaces of all $\xi^{(i)} \in L\left(K_{i}, X\right)$ such that $\xi^{(i)} \sqrt{Q^{(i)}}$ is a Hilbert-Schmidt operator with norm

$$
\left|\xi^{(i)}\right|_{L_{i}^{0}\left(K_{i}, X\right)}^{2}=\left|\xi^{(i)} \sqrt{Q^{(i)}}\right|_{\mathrm{HS}}^{2}=\operatorname{tr}\left(\xi^{(i)} Q^{(i)} \xi^{(i) *}\right)=\sum_{n=1}^{\infty}\left\|\sqrt{\lambda_{n}^{(i)}} \xi^{(i)} e_{n}^{i}\right\|_{X}^{2}<\infty
$$

such $\xi^{(i)}$ is called a $Q^{(i)}$-Hilbert-Schmidt operator, and the spaces $L_{i}^{0}\left(K_{i}, X\right)$ equipped with the inner product $\langle\phi, \psi\rangle_{L_{i}^{0}}=\sum_{n=1}^{\infty}\left\langle\phi e_{n}^{(i)}, \psi e_{n}^{(i)}\right\rangle$ are separable Hilbert spaces. Then there exist the real, independent standard Brownian motions sequences $\left\{w_{n}^{t}\right\}_{n \in N^{+}}$such that

$$
W(t)=\sum_{n=1}^{\infty} \sqrt{\lambda_{n}^{W}} e_{n}^{W} w_{n}(t), \quad t \geq 0
$$


The infinite dimensional cylindrical $K_{B}$-valued $B^{H}(t)$ is defined by

$$
B^{H}(t)=\sum_{n=1}^{\infty} \sqrt{\lambda_{n}^{B}} e_{n}^{B} \beta_{n}^{H}(t), \quad t \geq 0
$$

where $\left\{\beta_{n}^{H}(t)\right\}_{n \in N^{+}}$are real, independent fractional Brownian motions, we know $B^{H}(t)$ is a centered Gaussian process with covariance function

$$
R_{H}(s, t)=\frac{1}{2}\left(t^{2 H}+s^{2 H}-|t-s|^{2 H}\right) .
$$

Furthermore, $B^{H}(t)$ has the following Wiener integral:

$$
B^{H}(t)=\int_{0}^{t} K_{H}(t, s) d B(s)
$$

where $\{B(t), t \in[0,+\infty)\}$ is a standard Brownian motion, and $K_{H}(t, s)$ is the kernel,

$$
K_{H}(t, s)= \begin{cases}c_{H} s^{1 / 2-H} \int_{s}^{t}(u-s)^{H-3 / 2} u^{H-1 / 2} d u, & t>s, \\ 0, & t \leq s,\end{cases}
$$

where $c_{H}=\sqrt{\frac{H(2 H-1)}{\mathcal{B}\left(2-2 H, H-\frac{1}{2}\right)}}, \mathcal{B}(\cdot, \cdot)$ denotes the beta function.

The next lemmas are of prime importance to prove the existence and uniqueness of the mild solution of system (1). Lemma 2.1 and Lemma 2.3 give us a connection between stochastic integral and Riemann integral, and Lemma 2.2 is a bridge of $p$ th moment and mean-square moment of centered Gaussian random variable.

Lemma 2.1 ([34]) For any $p>0$ and $L_{W}^{0}$-valued process $\varphi(\cdot)$, there exists

$$
\sup _{s \in[0, t]} E\left\|\int_{0}^{s} \varphi(u) d W(u)\right\|_{X}^{2 p} \leq(p(2 p-1))^{p}\left(\int_{0}^{t}\left(E\|\varphi(s)\|_{L_{W}^{0}}^{2 p}\right)^{1 / p} d s\right)^{p}
$$

Lemma 2.2 ([35]) For arbitrary $T>0, \int_{0}^{T} \psi(s) d B^{H}(s)$ is a centered Gaussian random variable, for every $p>0$, there exists a constant $k(p)$ such that

$$
E\left\|\int_{0}^{T} \psi(s) d B^{H}(s)\right\|^{p} \leq k(p)\left(E\left\|\int_{0}^{T} \psi(s) d B^{H}(s)\right\|^{2}\right)^{\frac{p}{2}} .
$$

Lemma 2.3 ([36]) For any $\psi:[0,+\infty) \rightarrow L_{B}^{0}(Y, X)$ such that $\sum_{n=1}^{\infty}\left\|\psi Q^{\frac{1}{2}} e_{n}\right\|_{L^{1 / H}([0,+\infty) ; X)}<$ $\infty$ holds and for any $a, b \geq 0$,

$$
E\left\|\int_{a}^{b} \psi(s) d B^{H}(s)\right\|_{X}^{2} \leq c_{H} H(2 H-1)(b-a)^{2 H-1} \sum_{n=1}^{\infty} \int_{a}^{b}\left\|\psi(s) Q^{\frac{1}{2}} e_{n}\right\|_{X}^{2} d s
$$

moreover, if $\sum_{n=1}^{\infty}\left\|\psi(t) Q^{\frac{1}{2}} e_{n}\right\|_{X}$ is uniformly convergent for $t \in[0,+\infty)$, then

$$
E\left\|\int_{a}^{b} \psi(s) d B^{H}(s)\right\|_{X}^{2} \leq c_{H} H(2 H-1)(b-a)^{2 H-1} \int_{a}^{b}\|\psi(s)\|_{L_{B}^{0}}^{2} d s .
$$


We end this section by the introduction of some basic concepts about analytic semigroups and fractional powers of operators, for a comprehensive understanding of analytic semigroup theory we refer to [37]. If the dynamical system's present state is given by a point $\mathrm{x}$ in a Banach space $X$ and the future state at time $t$ is $T(t) x$, then the operators $\{T(t), t \geq 0\}$ in $X$ defines a semigroup of linear operators, the term semigroup arises from the property $T(t+s) x=T(t) T(s) x$, which means that the state at time $t+s$ starting at $x$ agrees with the state at time $t$ starting at $T(s) x$ (note that $T(0)=I$ ). Let $A: D(A) \rightarrow X$ be the infinitesimal generator of an analytic semigroup of bounded linear operators $(T(t))_{t \geq 0}$ on $X$. We assume that $(T(t))_{t \geq 0}$ is a uniformly bounded and analytic semigroup such that $0 \in \rho(A)$, where $\rho(A)$ is the resolvent set of $A$. Now we can define the fractional power $(-A)^{\alpha}$ for $0<\alpha \leq 1$ as a closed linear operator on its domain $D(-A)^{\alpha}$, which is dense in $X$ and the equality $\|\rho\|_{\alpha}=\left\|(-A)^{\alpha} \rho\right\|$ defines a norm in $D(-A)^{\alpha}$. Also $X_{\alpha}$ represents the space $D(-A)^{\alpha}$ endowed with the norm $\|\cdot\|_{\alpha}$.

Lemma 2.4 ([37]) Suppose that the above conditions are satisfied.

(1) If $0<\alpha \leq 1$, then $X_{\alpha}$ is a Banach space.

(2) If $0<\beta \leq \alpha$, then the injection $X_{\alpha} \hookrightarrow X_{\beta}$ is continuous.

(3) There exist $M_{\alpha}>0, \lambda>0$ such that

$$
\left\|(-A)^{\alpha} T(t)\right\| \leq \frac{M_{\alpha}}{t^{\alpha}} e^{-\lambda t}, \quad t>0
$$

for every $0<\alpha \leq 1$.

Definition 2.1 ([37]) A semigroup $T(t), t \geq 0$ of bounded linear operators on $X$ is strongly continuous if

$$
\lim _{t \rightarrow 0}(T(t)-I) x=0,
$$

for every $x \in X$.

\section{Existence and uniqueness of mild solution}

In this section, we obtain some new sufficient conditions to ensure the existence and uniqueness of mild solutions of system (1) by means of the Banach fixed point theorem, before proceeding, let us give the definition of a mild solution.

Definition 3.1 A $X$-valued stochastic process $x(t), t \in[-r, a]$ is called a mild solution of the Cauchy problem (1) if

(i) $x(t)=\phi(t)$, for $t \in[-r, 0]$.

(ii) $x(\cdot) \in \mathcal{P C}\left([-r, a], L^{p}(\Omega, X)\right)$ (the space of all functions that continuous everywhere except a finite number of points from $[-r, a]$ to $L^{p}(\Omega, X)$, provided with the supremum norm $\|\xi\|^{p}=\sup _{s \in[-r, a]}\left(E\|\xi(s)\|^{p}\right), 1<p<+\infty$.

(iii) For $t \in[0,+\infty), x(t)$ satisfies the following integral equation:

$$
\begin{aligned}
x(t)= & T(t)[\phi(0)-p(0, \phi)]+p\left(t, x_{t}\right)+\int_{0}^{t} A T(t-s) p\left(s, x_{s}\right) d s \\
& +\int_{0}^{t} T(t-s) f\left(s, x_{s}\right) d s
\end{aligned}
$$




$$
\begin{aligned}
& +\int_{0}^{t} T(t-s)\left(\int_{0}^{s} h\left(s, \eta, x_{\eta}\right) d \eta\right) d s+\int_{0}^{t} T(t-s) g\left(s, x_{s}\right) d W(s) \\
& +\int_{0}^{t} T(t-s) \sigma(s) d B^{H}(s)+\sum_{0<t_{k}<t} T\left(t-t_{k}\right) I_{k}\left(x\left(t_{k}^{-}\right)\right) \quad \mathbb{P} \text {-a.s. }
\end{aligned}
$$

To ensure the existence and the uniqueness of the above mild solution, we assume that the following hypotheses hold.

$(\mathrm{H} 1) \mathrm{A}$ is the infinitesimal generator of an analytic semigroup $(T(t))_{t \geq 0}$ of bounded linear operators on $X$ and satisfies $0 \in \sigma(A)$, together with Lemma 2.4, there exist constants $M, M_{1-\alpha}$ such that

$$
\|T(t)\| \leq M,\left\|(-A)^{1-\alpha} T(t)\right\| \leq \frac{M_{1-\alpha}}{t^{1-\alpha}} .
$$

(H2) For $\phi, \psi \in \mathcal{P C}$ and $t \in[0, a]$, there exist constants $\frac{1}{2}<\alpha<1$ and $L_{1}>0$ such that the mapping $p:[0,+\infty) \times \mathcal{P C} \rightarrow X$ satisfies the condition

$$
\left\|(-A)^{\alpha} p(t, \phi)-(-A)^{\alpha} p(t, \psi)\right\| \leq L_{1}\|\phi-\psi\|
$$

also, $(-A)^{\alpha} p$ is a continuous function in $p$ th moment sense

$$
\lim _{t \rightarrow s} E\left\|(-A)^{\alpha} p\left(t, x_{t}\right)-(-A)^{\alpha} p\left(s, x_{s}\right)\right\|^{p}=0
$$

(H3) For any $\phi, \psi \in \mathcal{P C}$ and $t \in[0, a]$, the mappings $f, h, g, I_{k}$ satisfy the following Lipschitz conditions, namely, there exist some constants $L_{2}, L_{3}, L_{4}, q_{k}\left(\sum_{k=1}^{\infty} q_{k}<\infty\right)$ such that

$$
\begin{aligned}
& \|f(t, \phi)-f(t, \psi)\| \leq L_{2}\|\phi-\psi\|, \\
& \left\|\int_{0}^{t} h(t, s, \phi)-h(t, s, \psi) d s\right\| \leq L_{3}\|\phi-\psi\|, \\
& \|g(t, \phi)-g(t, \psi)\|_{L_{W}^{0}} \leq L_{4}\|\phi-\psi\|, \\
& \left\|I_{k}(\phi)-I_{k}(\psi)\right\| \leq q_{k}\|\phi-\psi\|, \quad k=1,2, \ldots,
\end{aligned}
$$

for simplicity, we may take $f(t, 0)=h(t, s, 0)=g(t, 0)=I_{k}(0)=0$.

By employing the above hypotheses, we can establish the existence and uniqueness results for system (1).

Theorem 3.1 If Hypotheses (H1), (H2), (H3) hold and

$$
k+\left(\frac{5}{1-k}\right)^{p-1} M^{p}\left(\sum_{k=1}^{\infty} q_{k}\right)^{p}<1
$$

where $k=\left\|(-A)^{-\alpha}\right\| L_{1}, 1<p<\infty$, then system (1) has a unique mild solution.

Proof Fix $a>0$, we first introduce the set $\Lambda_{a}=\left\{\mathcal{P C}\left([-r, a], L^{p}(\Omega, X)\right)\right\}, 1<p<+\infty$, then define an operator $\pi$ as follows:

$$
(\pi x)(t)=T(t)[\phi(0)-p(0, \phi)]+p\left(t, x_{t}\right)+\int_{0}^{t} A T(t-s) p\left(s, x_{s}\right) d s
$$




$$
\begin{aligned}
& +\int_{0}^{t} T(t-s) f\left(s, x_{s}\right) d s \\
& +\int_{0}^{t} T(t-s)\left(\int_{0}^{s} h\left(s, \eta, x_{\eta}\right) d \eta\right) d s+\int_{0}^{t} T(t-s) g\left(s, x_{s}\right) d W(s) \\
& +\int_{0}^{t} T(t-s) \sigma(s) d B^{H}(s)+\sum_{0<t_{k}<t} T\left(t-t_{k}\right) I_{k}\left(x\left(t_{k}^{-}\right)\right), \quad t \in[0, a]
\end{aligned}
$$

and $(\pi x)(t)=\phi(t), t \in[-r, 0]$. Now our problem changes into a Banach fixed point problem, we will show the operator $\pi$ has a fixed point on interval $[-r, a]$. We divide our proof into two steps.

Step 1: It is obvious that the operator $\pi$ is continuous on $[-r, 0]$, now we will show it is continuous in $p$ th moment on $[0, a]$. Let $x \in \Lambda_{a}, 0<t<a$, and let $r>0$ be sufficiently small, then using the $C_{p}$ inequality: $E\left\|\sum_{i=1}^{n} x_{i}(t)\right\|^{p} \leq n^{p-1} \sum_{i=1}^{n} E\left\|x_{i}(t)\right\|^{p}$, where $p>1$ and $n>1$. we have

$$
\begin{aligned}
E \|( & \pi x)(t+r)-(\pi x)(t) \|^{p} \\
\leq & 8^{p-1}\left\{E\|(T(t+r)-T(t))(\phi(0)-p(0, \phi))\|^{p}+E\left\|p\left(t+r, x_{t+r}\right)-p\left(t, x_{t}\right)\right\|^{p}\right. \\
& +E\left\|\int_{0}^{t}(T(r)-I) A T(t-s) p\left(s, x_{s}\right) d s+\int_{t}^{t+r} A T(t+r-s) p\left(s, x_{s}\right) d s\right\|^{p} \\
& +E\left\|\int_{0}^{t}(T(r)-I) T(t-s) f\left(s, x_{s}\right) d s+\int_{t}^{t+r} T(t+r-s) f\left(s, x_{s}\right) d s\right\|^{p} \\
& +E \| \int_{0}^{t}(T(r)-I) T(t-s)\left(\int_{0}^{s} h\left(s, \eta, x_{\eta}\right) d \eta\right) d s \\
& +\int_{t}^{t+r} T(t+r-s)\left(\int_{0}^{s} h\left(s, \eta, x_{\eta}\right) d \eta\right) d s \|^{p} \\
& +E\left\|\int_{0}^{t}(T(r)-I) T(t-s) g\left(s, x_{s}\right) d W(s)+\int_{t}^{t+r} T(t+r-s) g\left(s, x_{s}\right) d W(s)\right\|^{p} \\
& +E\left\|\int_{0}^{t}(T(r)-I) T(t-s) \sigma(s) d B^{H}(s)+\int_{t}^{t+r} T(t+r-s) \sigma(s) d B^{H}(s)\right\|^{p} \\
& \left.+E\left\|\sum_{0<t_{k}<t}(T(r)-I) T\left(t-t_{k}\right) I_{k}\left(x\left(t_{k}^{-}\right)\right)+\sum_{0<t_{k}<t} T\left(t+r-t_{k}\right) I_{k}\left(x\left(t_{k}^{-}\right)\right)\right\|^{p}\right\} \\
:= & 8^{p-1} \sum_{i=1}^{8} E\left\|G_{i}(t+r)-G_{i}(t)\right\|^{p} .
\end{aligned}
$$

By virtue of the semigroup property and strong continuity of $T(t)$, we have

$$
\lim _{r \rightarrow 0}(T(t+r)-T(t))(\phi(0)-p(0, \phi))=\lim _{r \rightarrow 0}(T(r)-I) T(t)(\phi(0)-p(0, \phi))=0,
$$

by using Hypothesis (H1), we arrive at

$$
\|(T(r)-I) T(t)(\phi(0)-p(0, \phi))\| \leq 2 M^{2}\|(\phi(0)-p(0, \phi))\|,
$$


hence in terms of Lebesgue dominated convergence theory, we obtain

$$
E\left\|G_{1}(t+r)-G_{1}(t)\right\|^{p} \longrightarrow 0, \quad \text { as } r \longrightarrow 0 .
$$

Since the operator $(-A)^{-\alpha}$ is bounded and by Hypothesis $(\mathrm{H} 2)$ we get

$$
E\left\|G_{2}(t+r)-G_{2}(t)\right\|^{p} \longrightarrow 0, \quad \text { as } r \longrightarrow 0 .
$$

As for the third term, by using the $C_{p}$ inequality, we have

$$
\begin{aligned}
E\left\|G_{3}(t+r)-G_{3}(t)\right\|^{p} & \\
\leq & 2^{p-1} E\left\|\int_{0}^{t}(T(r)-I)(-A)^{1-\alpha} T(t-s)(-A)^{\alpha} p\left(s, x_{s}\right) d s\right\|^{p} \\
& +2^{p-1} E\left\|\int_{t}^{t+r}(-A)^{1-\alpha} T(t+r-s)(-A)^{\alpha} p\left(s, x_{s}\right) d s\right\|^{p} .
\end{aligned}
$$

It follows from Hypotheses $(\mathrm{H} 1)$ and $(\mathrm{H} 2)$ that

$$
\begin{aligned}
& \left\|(T(r)-I)(-A)^{1-\alpha} T(t-s)(-A)^{\alpha} p\left(s, x_{s}\right)\right\| \leq \frac{2 M M_{1-\alpha} L_{1}}{(t-s)^{1-\alpha}}\left\|x_{s}\right\|, \\
& \left\|(-A)^{1-\alpha} T(t+r-s)(-A)^{\alpha} p\left(s, x_{s}\right)\right\| \leq \frac{M_{1-\alpha} L_{1}}{(t+r-s)^{1-\alpha}}\left\|x_{s}\right\| .
\end{aligned}
$$

Thus, by Lebesgue dominated convergence theorem, we obtain

$$
E\left\|G_{3}(t+r)-G_{3}(t)\right\|^{p} \longrightarrow 0, \quad \text { as } r \longrightarrow 0 .
$$

By Hypotheses ( $\mathrm{H} 1)$ and $(\mathrm{H} 3)$, and using the same argument as in the proof of the third term, we can easily calculate that

$$
E\left\|G_{4}(t+r)-G_{4}(t)\right\|^{p} \longrightarrow 0, \quad \text { as } r \longrightarrow 0 .
$$

and

$$
E\left\|G_{5}(t+r)-G_{5}(t)\right\|^{p} \longrightarrow 0, \quad \text { as } r \longrightarrow 0 .
$$

Further, by using Lemma 2.1 and the $C_{p}$ inequality we have

$$
\begin{aligned}
& E\left\|G_{6}(t+r)-G_{6}(t)\right\|^{p} \\
& \leq 2^{p-1} C(p)\left(\int_{0}^{t}\left(E\left\|(T(r)-I) T(t-s) g\left(t, x_{s}\right)\right\|_{L_{W}^{0}}^{p}\right)^{\frac{2}{p}} d s\right)^{\frac{p}{2}} \\
& \quad+2^{p-1} C(p)\left(\int_{t}^{t+r}\left(E\left\|T(t+r-s) g\left(t, x_{s}\right)\right\|_{L_{W}^{0}}^{p}\right)^{\frac{2}{p}} d s\right)^{\frac{p}{2}},
\end{aligned}
$$

where $C(p)=(p(p-1) / 2)^{p / 2}$. 
By Hypotheses (H1) and (H3), the right hand side of the above inequality becomes

$$
\left\|(T(r)-I) T(t-s) g\left(t, x_{s}\right)\right\|_{L_{W}^{0}} \leq 2 M^{2} L_{4}\left\|x_{s}\right\|
$$

and

$$
\left\|T(t+r-s) g\left(s, x_{s}\right)\right\|_{L_{W}^{0}} \leq M L_{4}\left\|x_{s}\right\|
$$

Applying Lebesgue dominated convergence theorem, we conclude that

$$
E\left\|G_{6}(t+r)-G_{6}(t)\right\|^{p} \longrightarrow 0, \quad \text { as } r \longrightarrow 0 .
$$

Now we consider the seventh term, by using the $C_{p}$ inequality, we have

$$
\begin{aligned}
E\left\|G_{7}(t+r)-G_{7}(t)\right\|^{p} \leq & 2^{p-1} E\left\|\int_{0}^{t}(T(r)-I) T(t-s) \sigma(s) d B^{H}(s)\right\|^{p} \\
& +2^{p-1} E\left\|\int_{t}^{t+r} T(t+r-s) \sigma(s) d B^{H}(s)\right\|^{p} \\
:= & K 1+K 2 .
\end{aligned}
$$

By virtue of Lemma 2.2 and Lemma 2.3, respectively, together with Hypothesis (H1) we arrive at

$$
\begin{aligned}
K 1 & \leq 2^{p-1} k(p) E\left(\left\|\int_{0}^{t}(T(r)-I) T(t-s) \sigma(s) d B^{H}(s)\right\|^{2}\right)^{\frac{p}{2}} \\
& \leq 2^{p-1} M^{p} k(p)\left(c_{H} H(2 H-1) t^{2 H-1}\right)^{\frac{p}{2}}\left(\int_{0}^{t}\|(T(r)-I) \sigma(s)\|_{L_{B}^{0}}^{2} d s\right)^{\frac{p}{2}} \rightarrow 0 \quad \text { as } r \rightarrow 0,
\end{aligned}
$$

this is because $T(r) \sigma(s) \rightarrow \sigma(s)$ as $r \rightarrow 0$ and $\|T(r) \sigma(s)\|_{L_{B}^{0}} \leq M\|\sigma(s)\|_{L_{B}^{0}}$ for any $s>0$.

Similarly, we have

$$
K 2 \leq 2^{p-1} M^{p} k(p)\left(c_{H} H(2 H-1) r^{2 H-1}\right)^{\frac{p}{2}}\left(\int_{t}^{t+r}\|\sigma(s)\|_{L_{B}^{0}}^{2} d s\right)^{\frac{p}{2}} \longrightarrow 0, \quad \text { as } r \longrightarrow 0 .
$$

Hence

$$
E\left\|G_{7}(t+r)-G_{7}(t)\right\|^{p} \longrightarrow 0, \quad \text { as } r \longrightarrow 0 .
$$

As for the last term, by the $C_{p}$ inequality, we have

$$
\begin{aligned}
E\left\|G_{8}(t+r)-G_{8}(t)\right\|^{p} \leq & 2^{p-1} E\left\|\sum_{0<t_{k}<t}(T(r)-I) T\left(t-t_{k}\right) I_{k}\left(x\left(t_{k}^{-}\right)\right)\right\|^{p} \\
& +2^{p-1} E\left\|\sum_{t<t_{k}<t+r} T\left(t+r-t_{k}\right) I_{k}\left(x\left(t_{k}^{-}\right)\right)\right\|^{p}
\end{aligned}
$$


By Hypothesis (H3) we get

$$
\begin{aligned}
& \left\|(T(r)-I) T\left(t-t_{k}\right) I_{k}\left(x\left(t_{k}^{-}\right)\right)\right\| \leq\left\|2 M^{2} q_{k}\right\| x\left(t_{k}^{-}\right) \|, \\
& \left\|T\left(t+r-t_{k}\right) I_{k}\left(x\left(t_{k}^{-}\right)\right)\right\| \leq M q_{k}\left\|x\left(t_{k}^{-}\right)\right\| .
\end{aligned}
$$

Thus from the Lebesgue dominated convergence theorem, we have

$$
E\left\|G_{8}(t+r)-G_{8}(t)\right\|^{p} \longrightarrow 0, \quad \text { as } r \longrightarrow 0 .
$$

The combination of all the estimations of $E\left\|G_{i}(t+r)-G_{i}(t)\right\|^{p}, i=1,2, \ldots, 8$, yields

$$
\lim _{r \rightarrow 0} E\|(\pi x)(t+r)-(\pi x)(t)\|^{p}=0 .
$$

Hence, we conclude that the mapping $\pi$ is continuous in $p$ th moment on $[-r, a]$.

Step 2: We show the mapping $\pi$ is a contraction.

By using the $C_{p}$ inequality and the elementary inequality $|u+v|^{p} \leq \frac{|u|^{p}}{k^{p-1}}+\frac{|v|^{p}}{(1-k)^{p-1}}$ for $u, v \in X$ and $k \in(0,1)$, we get

$$
\begin{aligned}
E\|(\pi x)(t)-(\pi y)(t)\|^{p} & \\
\leq & k^{1-p} E\left\|p\left(t, x_{t}\right)-p\left(t, y_{t}\right)\right\|^{p} \\
& +\left(\frac{5}{1-k}\right)^{p-1} E\left\{\left\|\int_{0}^{t} A T(t-s)\left(p\left(s, x_{s}\right)-p\left(s, y_{s}\right)\right) d s\right\|^{p}\right. \\
& +\left\|\int_{0}^{t} T(t-s)\left(f\left(s, x_{s}\right)-f\left(s, y_{s}\right)\right) d s\right\|^{p} \\
& +\left\|\int_{0}^{t} T(t-s)\left[\int_{0}^{s}\left(h\left(s, \eta, x_{\eta}\right)-h\left(s, \eta, y_{\eta}\right)\right) d \eta\right] d s\right\|^{p} \\
& +\left\|\int_{0}^{t} T(t-s)\left(g\left(s, x_{s}\right)-g\left(s, y_{s}\right)\right) d w(s)\right\|^{p} \\
& \left.+\left\|\sum_{0<t_{k}<t} T\left(t-t_{k}\right)\left[I_{k}\left(x\left(t_{k}^{-}\right)\right)-I_{k}\left(y\left(t_{k}^{-}\right)\right)\right]\right\|^{p}\right\} \\
:= & k^{1-p} F 1+\left(\frac{5}{1-k}\right)^{p-1} \sum_{i=2}^{6} F i .
\end{aligned}
$$

From Hypothesis (H2), we obtain

$$
F 1 \leq\left\|(-A)^{-\alpha}\right\|^{p} L_{1}^{p} E\left\|x_{t}-y_{t}\right\|^{p} \leq k^{p} \sup _{s \in[-r, t]} E\|x(s)-y(s)\|^{p}
$$

By Hypotheses (H1) and (H2) and the Hölder inequality, we get

$$
\begin{aligned}
F 2 & =E\left\|\int_{0}^{t}(-A)^{1-\alpha} T(t-s)(-A)^{\alpha}\left(p\left(s, x_{s}\right)-p\left(y, y_{s}\right)\right) d s\right\|^{p} \\
& \leq\left[\int_{0}^{t} M_{1-\alpha}^{q}(t-s)^{q \alpha-q} d s\right]^{p-1} E \int_{0}^{t} L_{1}^{p}\left\|x_{s}-y_{s}\right\|^{p} d s
\end{aligned}
$$




$$
\leq \frac{M_{1-\alpha}^{p} L_{1}^{p} t^{p \alpha}}{(1+q \alpha-q)^{p-1}} \sup _{s \in[-r, t]} E\|x(s)-y(s)\|^{p}
$$

where $1<p<\infty, \frac{1}{p}+\frac{1}{q}=1$.

Using Hypotheses (H1) and (H3), we have

$$
\begin{aligned}
F 3 & \leq\left(\int_{0}^{t} M L_{2}\left\|x_{s}-y_{s}\right\| d s\right)^{p} \\
& \leq M^{p} L_{2}^{p} t^{p} \sup _{s \in[-r, t]}\|x(s)-y(s)\|^{p} .
\end{aligned}
$$

Similarly, by Hypotheses (H1), (H3) we have

$$
F 4 \leq M^{p} L_{3}^{p} t^{p} \sup _{s \in[-r, t]}\|x(s)-y(s)\|^{p} .
$$

As for the fifth term, by Lemma 2.1 and Hypothesis (H3) we can arrive at

$$
\begin{aligned}
F 5 & \leq C(p)\left\{\int_{0}^{t}\left[E\left\|T(t-s)\left(g\left(s, x_{s}\right)-g\left(s, y_{s}\right)\right)\right\|_{L_{w}^{0}}^{p}\right]^{\frac{2}{p}} d s\right\}^{\frac{p}{2}} \\
& \leq C(p) E\left\{\int_{0}^{t}\left[M^{p} L_{4}^{p} E\left\|x_{s}-y_{s}\right\|^{p}\right]^{\frac{2}{p}} d s\right\}^{\frac{p}{2}} \\
& \leq C(p) M^{p} L_{4}^{p} t^{\frac{p}{2}} \sup _{s \in[-r, t]}\|x(s)-y(s)\|^{p},
\end{aligned}
$$

where $C(p)=(p(p-1) / 2)^{p / 2}$.

From Hypothesis (H1), (H3) and the Hölder inequality, we get

$$
\begin{aligned}
F 6 & =E\left\{\sum_{k=1}^{\infty}\left\|T\left(t-t_{k}\right)\left[I_{k}\left(x\left(t_{k}^{-}\right)\right)-I_{k}\left(y\left(t_{k}^{-}\right)\right)\right]\right\|\right\}^{p} \\
& \leq M^{p} E\left\{\sum_{k=1}^{\infty} q_{k}\left\|x\left(t_{k}^{-}\right)-y\left(t_{k}^{-}\right)\right\|\right\}^{p} \\
& =M^{p} E\left\{\sum_{k=1}^{\infty} q_{k}^{\frac{1}{q}} q_{k}^{\frac{1}{p}}\left\|x\left(t_{k}^{-}\right)-y\left(t_{k}^{-}\right)\right\|\right\}^{p} \\
& \leq M^{p} E\left\{\left(\sum_{k=1}^{\infty} q_{k}\right)^{p-1} \sum_{k=1}^{\infty} q_{k}\left\|x\left(t_{k}^{-}\right)-y\left(t_{k}^{-}\right)\right\|^{p}\right\} \\
& =M^{p}\left(\sum_{k=1}^{\infty} q_{k}\right)^{p} E\left\|x\left(t_{k}^{-}\right)-y\left(t_{k}^{-}\right)\right\|^{p} \\
& \leq M^{p}\left(\sum_{k=1}^{\infty} q_{k}\right)^{p} \sup _{s \in[-r, t]} E\|x(s)-y(s)\|^{p} .
\end{aligned}
$$

Substituting (6)-(11) into (5) yields

$$
\sup _{s \in[-r, t]} E\|(\pi x)(s)-(\pi y)(s)\|^{p} \leq \rho(t) \sup _{s \in[-r, t]} E\|x(s)-y(s)\|^{p},
$$


where

$$
\begin{aligned}
\rho(t)= & k+\left(\frac{5}{1-k}\right)^{p-1}\left[\frac{M_{1-\alpha}^{p} L_{1}^{p} t^{p \alpha}}{(1+q \alpha-q)^{p-1}}+M^{p}\left(L_{2}^{p}+L_{3}^{p}\right) t^{p}\right. \\
& \left.+C(p) M^{p} L_{4}^{p} t^{\frac{p}{2}}+M^{p}\left(\sum_{k=1}^{\infty} q_{k}\right)^{p}\right] .
\end{aligned}
$$

Thus, it follows from inequality(3) that

$$
\rho(0)=k+\left(\frac{5}{1-k}\right)^{p-1} M^{p}\left(\sum_{k=1}^{\infty} q_{k}\right)^{p}<1 .
$$

Then there exists $0<a_{1} \leq a$ such that $0<\rho\left(a_{1}\right)<1$, so the operator $\pi$ is a contraction on $\Lambda_{a_{1}}$ and thus it has a unique fixed point on $\left[-r, a_{1}\right]$, which is a mild solution of system (1). Obviously, this process can be repeated for the entire interval $[-r, a]$ in finitely many steps, this completes the proof.

\section{Exponential stability in the $p$ th moment}

In this section, we establish some sufficient conditions to ensure the exponential stability in $p$ th moment of a mild solution for system (1). Before proceeding, let us introduce the following additional hypotheses and Definition 4.1.

(H4) We suppose that the semigroup $T(t)$ is exponentially stable, i.e., there exist $\lambda>0$ and $M>0$ such that $\|T(t)\| \leq M e^{-\lambda t}, \forall t \geq 0$.

(H5) The mapping $\sigma:[0,+\infty) \mapsto L_{B}^{0}(Y, X)$ satisfies

$$
\int_{0}^{+\infty} e^{\lambda s}\|\sigma(s)\|_{L_{B}^{0}}^{p} d s<\infty
$$

Definition 4.1 ([14]) Let $p$ be an integer $p \geq 2$. The mild solution of system (1) is said to be exponentially stable in the $p$ th moment if, for any initial value $\phi$, there exist a pair of constants $\alpha>0$ and $C>0$ such that

$$
E\|x(t)\|^{p} \leq C e^{-\alpha t}, \quad t \geq 0 .
$$

In particular, if $p=2$, then system (1) is said to be mean-square exponentially stable.

The next lemma is essential to the proof of exponential stability in $p$ th moment of mild solution of system (1), for a brief proof we refer to [38].

Lemma 4.1 ([38]) For any $\mu>0$, suppose that there exist some positive constants $\alpha_{i}$ (i= $1,2,3), \beta_{k}(k-1,2, \ldots, m)$ and a function $\phi:[-r, \infty) \mapsto[0, \infty)$ such that

$$
\left\{\begin{aligned}
\phi(t) \leq & \alpha_{1} e^{-\mu t}, \quad t \in[-r, 0), \\
\phi(t) \leq & \alpha_{1} e^{-\mu t}+\alpha_{2} \sup _{\theta \in[-r, 0]} \phi(t+\theta)+\alpha_{3} \int_{0}^{t} e^{-\mu(t-s)} \sup _{\theta \in[-r, 0]} \phi(s+\theta) d s \\
& +\sum_{t_{k}<t} \beta_{k} e^{-\mu\left(t-t_{k}\right)} \phi\left(t_{k}^{-}\right), \quad t \in[0, \infty) .
\end{aligned}\right.
$$


If $\alpha_{2}+\frac{\alpha_{3}}{\mu}+\sum_{k=1}^{m} \beta_{k}<1$, then there exist constants $N>0$ and $\mu>0$ such that $\phi(t) \leq N e^{-\mu t}$ for $t \geq-r$.

Now we state our results to the exponential stability of mild solution of system (1).

Theorem 4.1 Assume that Hypotheses (H1)-(H5) hold. Then the mild solution of system (1) is exponentially stable in the pth moment if

$$
\begin{aligned}
& 7^{p-1}\left\{M_{1-\alpha}^{p} \lambda^{-p \alpha} L_{1}^{p} \Gamma^{p-1}(1+q \alpha-q)+M^{p} \lambda^{-p}\left(L_{2}^{p}+L_{3}^{p}\right)\right. \\
& \left.+C(p) M^{p} L_{4}^{p} \lambda^{-\frac{p}{2}}\left(\frac{2 p-2}{p-2}\right)^{1-\frac{p}{2}}+M^{p}\left(\sum_{0<t_{k}<t} q_{k}\right)^{p}\right\}<(1-k)^{p}
\end{aligned}
$$

holds, where $C(p)=(p(p-1) / 2)^{p / 2}, k=\left\|(-A)^{-\alpha}\right\| L_{1}$.

Proof From inequality (12), we can find a number $\epsilon>0$ small enough and suppose that $\mu=\lambda-\epsilon$ satisfies the following inequality:

$$
\begin{aligned}
k & +\frac{7^{p-1}}{\mu(1-k)^{p-1}}\left\{M_{1-\alpha}^{p} \lambda^{1-p \alpha} L_{1}^{p} \Gamma^{p-1}(1+q \alpha-q)+M^{p} \lambda^{1-p}\left(L_{2}^{p}+L_{3}^{p}\right)\right. \\
& \left.+C(p) M^{p} L_{4}^{p}\left(\frac{2 \lambda p-2 \lambda}{p-2}\right)^{1-\frac{p}{2}}\right\}+\left(\frac{7}{1-k}\right)^{p-1} M^{p}\left(\sum_{0<t_{k}<t} L_{k}\right)^{p}<1 .
\end{aligned}
$$

From Eq. (2), by the $C_{p}$ inequality and the inequality $|u+v|^{p} \leq \frac{|u|^{p}}{k^{p-1}}+\frac{|v|^{p}}{(1-k)^{p-1}}$, we get

$$
\begin{aligned}
E\|x(t)\|^{p} \leq & k^{1-p} E\left\|p\left(t, x_{t}\right)\right\|^{p}+\left(\frac{7}{1-k}\right)^{p-1} E\left\{\|T(t)[\phi(0)-p(0, \phi)]\|^{p}\right. \\
& +\left\|\int_{0}^{t} T(t-s) p\left(s, x_{s}\right) d s\right\|^{p}+\left\|\int_{0}^{t} T(t-s) f\left(s, x_{s}\right) d s\right\|^{p} \\
& +\left\|\int_{0}^{t} T(t-s)\left(\int_{0}^{s} h\left(s, \eta, x_{\eta}\right) d \eta\right) d s\right\|^{p}+\left\|\int_{0}^{t} T(t-s) g\left(s, x_{s}\right) d W(s)\right\|^{p} \\
& \left.+\left\|\int_{0}^{t} T(t-s) \sigma(s) d B^{H}(s)\right\|^{p}+\left\|\sum_{0<t_{k}<t} T\left(t-t_{k}\right) I_{k}\left(x\left(t_{k}^{-}\right)\right)\right\|^{p}\right\} \\
:= & k^{1-p} J_{1}+\left(\frac{7}{1-k}\right)^{p-1} \sum_{i=2}^{8} J_{i} .
\end{aligned}
$$

It follows from Hypothesis (H2) that

$$
J_{1} \leq\left\|(-A)^{-\alpha}\right\|^{p} L_{1}^{p} E\left\|x_{t}\right\|^{p} \leq k^{p} \sup _{\theta \in[-r, 0]}\|x(s+\theta)\|^{p} .
$$

By Hypotheses (H2) and (H4) we get

$$
\begin{aligned}
J_{2} & \leq M^{p} e^{-\lambda p t}\left(2^{p-1} E\|\phi(0)\|^{p}+2^{p-1}\left\|(-A)^{-\alpha}\right\|^{p} L_{1}^{p} E\|\phi\|^{p}\right) \\
& \leq 2^{p-1} M^{p}\left(E\|\phi(0)\|^{p}+\left\|(-A)^{-\alpha}\right\|^{p} L_{1}^{p} E\|\phi\|^{p}\right) e^{-\mu t}
\end{aligned}
$$




$$
:=C_{1} e^{-\mu t} .
$$

Combining Hypotheses (H2) and Lemma 2.4 with the Hölder inequality, noting that $\left(\int_{0}^{t} e^{-\lambda(t-s)} d s\right)^{\frac{p}{q}} \leq\left(\frac{1}{\lambda}\right)^{p-1}$, we obtain

$$
\begin{aligned}
J_{3} & =E\left\|\int_{0}^{t}(-A)^{1-\alpha} T(t-s)(-A)^{\alpha} p\left(s, x_{s}\right) d s\right\|^{p} \\
& \leq M_{1-\alpha}^{p} L_{1}^{p}\left(\int_{0}^{t} e^{-\lambda(t-s)}(t-s)^{\alpha q-q} d s\right)^{p-1} \times \int_{0}^{t} e^{-\lambda(t-s)} E\left\|x_{s}\right\|^{p} d s \\
& \leq M_{1-\alpha}^{p} \lambda^{1-p \alpha} L_{1}^{p} \Gamma^{p-1}(1+q \alpha-q) \times \int_{0}^{t} e^{-\lambda(t-s)} \sup _{\theta \in[-r, 0]} E\|x(s+\theta)\|^{p} d s .
\end{aligned}
$$

Similarly, from Hypotheses (H3) and (H4) and the Hölder inequality we have

$$
\begin{aligned}
& J_{4} \leq M^{p} L_{2}^{p} \lambda^{1-p} \int_{0}^{t} e^{-\lambda(t-s)} \sup _{\theta \in[-r, 0]} E\|x(s+\theta)\|^{p} d s, \\
& J_{5} \leq M^{p} L_{3}^{p} \lambda^{1-p} \int_{0}^{t} e^{-\lambda(t-s)} \sup _{\theta \in[-r, 0]} E\|x(s+\theta)\|^{p} d s .
\end{aligned}
$$

By virtue of Lemma 2.1, Hypotheses (H3) and (H4) and the Hölder inequality, we get

$$
\begin{aligned}
J_{6} & \leq C(p) M^{p} L_{4}^{p}\left(\int_{0}^{t} e^{-2 \lambda(t-s)}\left(E\left\|x_{s}\right\|^{p}\right)^{\frac{2}{p}} d s\right)^{\frac{p}{2}} \\
& \leq C(p) M^{p} L_{4}^{p}\left(\frac{2 \lambda p-2 \lambda}{p-2}\right)^{1-\frac{p}{2}} \int_{0}^{t} e^{-\lambda(t-s)} \sup _{\theta \in[-r, 0]} E\|x(s+\theta)\|^{p} d s .
\end{aligned}
$$

As for the seventh term, using Lemma 2.2 and Lemma 2.3 successively, together with Hypothesis (H4) and the Hölder inequality, we obtain

$$
\begin{aligned}
J_{7} & \leq k(p) E\left(\left\|\int_{0}^{t} T(t-s) \sigma(s) d B^{H}(s)\right\|^{2}\right)^{\frac{p}{2}} \\
& \leq k(p)\left(C_{H} H(2 H-1) t^{2 H-1}\right)^{\frac{p}{2}}\left(\int_{0}^{t}\|T(t-s) \sigma(s)\|_{L_{B}^{0}}^{2} d s\right)^{\frac{p}{2}} \\
& \leq M^{p} k(p)\left(C_{H} H(2 H-1) t^{2 H-1}\right)^{\frac{p}{2}}\left(\frac{2 p \lambda-2 \lambda}{p-2}\right)^{1-\frac{p}{2}} e^{-\mu t} e^{-\epsilon t} \int_{0}^{t} e^{\lambda s}\|\sigma(s)\|_{L_{B}^{0}}^{p} d s,
\end{aligned}
$$

and thanks to Hypothesis (H5) there exists a positive constant $C_{2}>0$, for all $t \geq 0$ such that

$$
M^{p} k(p)\left(C_{H} H(2 H-1) t^{2 H-1}\right)^{\frac{p}{2}}\left(\frac{2 p \lambda-2 \lambda}{p-2}\right)^{1-\frac{p}{2}} e^{-\epsilon t} \int_{0}^{t} e^{\lambda s}\|\sigma(s)\|_{L_{B}^{0}}^{p} d s<C_{2}
$$

so we have

$$
J_{7} \leq C_{2} e^{-\mu t} .
$$


From Hypotheses (H3) and (H4) and the Hölder inequality, we obtain

$$
J_{8} \leq M^{p}\left(\sum_{0<t_{k}<t} q_{k}\right)\left(\sum_{0<t_{k}<t} q_{k}\right)^{p-1} e^{-\mu\left(t-t_{k}\right)} E\left\|x\left(t_{k}^{-}\right)\right\|^{p} .
$$

Thus, by inequalities (15)-(22) we have

$$
\begin{aligned}
E\|x(t)\|^{p} \leq & \left(\frac{7}{1-k}\right)^{p-1}\left(C_{1}+C_{2}\right) e^{-\mu t}+k \sup _{\theta \in[-r, 0]} E\|x(t+\theta)\|^{p} \\
& +\left(\frac{7}{1-k}\right)^{p-1}\left(M_{1-\alpha}^{p} \lambda^{1-p \alpha} L_{1}^{p} \Gamma^{p-1}(1+q \alpha-q)+M^{p} \lambda^{1-p}\left(L_{2}+L_{3}\right)\right. \\
& \left.+C(p) M^{p} L_{4}^{p}\left(\frac{2 \lambda p-2 \lambda}{p-2}\right)^{1-p / 2}\right) \int_{0}^{t} e^{-\mu(t-s)} \sup _{\theta \in[-r, 0]}\|x(s+\theta)\|^{p} d s \\
& +M^{p}\left(\sum_{0<t_{k}<t} q_{k}\right)^{p} e^{-\mu\left(t-t_{k}\right)} E\left\|x\left(t_{k}^{-}\right)\right\|^{p} .
\end{aligned}
$$

According to Lemma 4.1 and inequality (13), it follows that there exist constants $N>0$ and $\mu>0$ such that $E\|x(t)\|^{p} \leq N e^{-\mu t}$ for all $t \geq-r$. Then the proof is completed.

Corollary 4.1 Suppose that Hypotheses (H1)-(H5) hold with $p=2$. Then the mild solution of system (1) is exponentially stable in mean square provided that

$$
7\left\{M_{1-\alpha}^{2} L_{1}^{2} \Gamma(2 \alpha-1) \lambda^{-2 \alpha}+M^{2}\left(L_{2}^{2}+L_{3}^{2}\right) \lambda^{-2}+M^{2} L_{4}^{2} \lambda^{-1}+M^{2}\left(\sum_{0<t_{k}<t} q_{k}\right)^{2}\right\}<(1-k)^{2},
$$

where $k=\left\|(-A)^{-\alpha}\right\| L_{1}$.

Remark 4.1 In [26], Ren et al. considered the existence and uniqueness of the mild solution for a similar system, but they did not investigate the exponential stability.

Remark 4.2 When $\int_{0}^{t} h\left(t, x, x_{s}\right) d s=0$ and the impulsive items $\Delta x\left(t_{k}\right)=I_{k}(\cdot)=0, k=1,2, \ldots$ then the system (1) reduces to a NSFPDE driven by mixed fractional Brownian motion, which is studied by Zhang and Ruan [14]. By utilizing the same technique as in our paper, we can easily address the sufficient conditions to ensure the existence, uniqueness and exponential stability in $p$ th moment of mild solution. In this sense, our work generalized the results in [14].

Remark 4.3 When $p=2$ and the Brownian motion $W(t)$ is absent, then the system (1) reduces to a NSFPDE driven by fractional Brownian motion with impulsive effects, in which the mean-square exponential stability of mild solution has been studied by Ma et al. [23]. Obviously, the given result in [23] can be seen as a special case of the result of this paper. In this sense, the exponential stability result in this paper is a generalization of the result in [23].

Remark 4.4 In [9], the authors considered the existence and uniqueness of delayed impulsive stochastic Volterra integro-differential equation driven by $\mathrm{fBm}$ in a Hilbert space, 
the impulses in [9] are linear and delayed impulses, which not only depend on the current states but also on historical states. But they did not consider the neutral term and the standard Brownian motion.

Remark 4.5 In [24], the authors studied the stability of delayed impulsive stochastic differential equations driven by a fBm with time-varying delay, the impulses are delayed impulses, we would like to mention that our method used in Theorem 4.1 is also valid in the case of delayed impulses, but the process is very tedious, here we omit the details.

Remark 4.6 The results obtained in this manuscript can be generalized to a class of impulsive stochastic neutral systems, for instance, we could add a distributed delay term to the left hand side of system (1), namely, system (1) extends to the following form

$$
\left\{\begin{aligned}
d[x(t) & \left.-p\left(t, x_{t}\right)-\int_{0}^{t} q\left(s, x_{s}\right) d s\right] \\
= & {\left[A x(t)+f\left(x, x_{t}\right)\right.} \\
& \left.+\int_{0}^{t} h\left(t, s, x_{s}\right) d s\right] d t+g\left(t, x_{t}\right) d W(t) \\
& +\sigma(t) d B^{H}(t), \quad t \in[0,+\infty), t \neq t_{k}, \\
\Delta x\left(t_{k}\right)=I_{k}\left(x\left(t_{k}^{-}\right)\right), \quad t=t_{k}, k=1,2, \ldots, & \\
x(t)= & \phi(t) \in \mathcal{P C}([-r, 0] ; X), \quad t \in[-r, 0] .
\end{aligned}\right.
$$

Here the function $q:[0,+\infty) \times \mathcal{P C} \rightarrow X$ is integrable. By using the same method as in this manuscript, we could study the existence, uniqueness and exponential stability of a mild solution of system (23).

\section{Example}

In the present section, we give an example as an application of the obtained results. Let $X=Y=L^{2}[0, \pi]$ and $A=\frac{\partial_{2}}{\partial \tau^{2}}$ with domain $D(A)=H_{0}^{1}(0, \pi) \cap H^{2}(0, \pi)$. Then there exists a complete orthonormal set $e_{n}(n \in N)$ of eigenvectors of $A$ with $e_{n}(x)=\sqrt{2 / \pi} \sin n x(n \in N)$, and

$$
A x=-\sum_{n=1}^{+\infty} n^{2}\left\langle x, e_{n}\right\rangle e_{n}, \quad x \in D(A)
$$

is the infinitesimal generator of the semigroup $T(t)(t \geq 0)$ in $X, T(t)$ is

$$
T(t) x=\sum_{n=1}^{+\infty} e^{-n^{2} t}\left\langle x, e_{n}\right\rangle e_{n}, \quad x \in X .
$$

We know that $\|T(t)\| \leq e^{-\pi^{2} t}, t \geq 0$, the bounded linear operator $(-A)^{\frac{3}{4}}$ is defined by

$$
(-A)^{\frac{3}{4}} x=\sum_{n=1}^{+\infty} n^{\frac{3}{2}}\left\langle x, e_{n}\right\rangle_{X} e_{n}
$$

with domain

$$
D\left((-A)^{\frac{3}{4}}\right)=\left\{x \in X, \sum_{n=1}^{+\infty} n^{\frac{3}{2}}\left\langle x, e_{n}\right\rangle_{X} e_{n} \in X\right\} .
$$


Now we consider the following impulsive neutral stochastic integro-differential equation with delays:

$$
\left\{\begin{array}{l}
d\left[x(t, \tau)-\beta_{1}(t, x(t-\zeta, \tau))\right] \\
\quad=\quad\left[\frac{\partial^{2}}{\partial \tau^{2}} x(t, \tau)+\beta_{2}(t, x(t-\zeta, \tau))\right. \\
\left.\quad+\int_{0}^{s} \beta_{3}(t, s, x(s-\zeta, \tau)) d s\right] d t+\beta_{4}(t, x(t-\zeta, \tau)) d W(t) \\
\quad+\sigma(t) d B^{H}(t), \quad t \in[0, a], t \neq t_{k}, \\
x(t, 0)=x(t, \pi)=0, \\
\begin{array}{l}
\Delta x\left(t_{k}, \cdot\right)(\tau)=\frac{\tilde{\beta}_{5}}{k^{2}} x\left(t_{k}^{-}, \tau\right), \quad t=t_{k}, k=1,2, \ldots, \\
x(t, \tau)=\phi(t, \tau) \in \mathcal{P C}\left([-r, 0], L^{2}[0, \pi]\right), \quad t \in[-r, 0], r \geq 0,
\end{array}
\end{array}\right.
$$

where $\zeta \in(-\infty, t]$ and $\sigma:[0,+\infty) \rightarrow L_{B}^{0}(Y, X)$ is a continuous function satisfying Hypothesis $(\mathrm{H} 5)$.

Let

$$
\begin{aligned}
& \beta_{1}(t, x(t-\zeta, \tau)) \leq \tilde{\beta}_{1} x(t-\zeta, \tau), \\
& \beta_{2}(t, x(t-\zeta, \tau)) \leq \tilde{\beta}_{2} x(t-\zeta, \tau), \\
& \int_{0}^{s} \beta_{3}(t, s, x(s-\zeta, \tau)) d s \leq \tilde{\beta}_{3} x(t-\zeta, \tau), \\
& \beta_{4}(t, x(t-\zeta, \tau)) \leq \tilde{\beta}_{4} x(t-\zeta, \tau),
\end{aligned}
$$

where $\tilde{\beta}_{i}>0(i=1,2,3,4)$ are all constants.

It is apparent that all the hypotheses are satisfied with

$$
\begin{aligned}
& \lambda=\pi^{2}, \quad M=1, \quad r=1, \quad L_{1}=\tilde{\beta}_{1} \|(-A)^{\frac{3}{4} \|} \\
& L_{i}=\tilde{\beta}_{i} \quad(i=2,3,4), \quad q_{k}=\frac{\tilde{\beta}_{5}}{k^{2}} \quad(k=1,2,3, \ldots) .
\end{aligned}
$$

From the definition of a bounded linear operator $(-A)^{-\frac{3}{4}}$ (see [37]), we obtain

$$
\left\|(-A)^{-\frac{3}{4}}\right\| \leq \frac{1}{\Gamma\left(\frac{3}{4}\right)} \int_{0}^{+\infty} t^{-\frac{1}{4}}\|T(t)\| d t \leq \frac{1}{\pi^{\frac{3}{2}}}
$$

and $\left\|(-A)^{\frac{3}{4}}\right\|=1$.

Consequently, by Theorem 3.1, we can deduce that if

$$
\frac{5 \pi^{2}}{36}<\left(1-\frac{\tilde{\beta}_{1}}{\pi^{\frac{3}{2}}}\right)^{2}
$$

then system (24) has a unique mild solution (here $p=2$ and $\left.k=\left\|(-A)^{-\frac{3}{4}}\right\| \tilde{\beta}_{1}\right)$. Moreover, by virtue of Corollary 4.1, the mild solution of system (24) is exponentially stable in meansquare moment if the following inequality holds:

$$
7\left(\frac{\tilde{\beta}_{1}^{2}}{\pi^{\frac{5}{2}}}+\frac{\tilde{\beta}_{2}^{2}+\tilde{\beta}_{3}^{2}}{\pi^{4}}+\frac{\tilde{\beta}_{4}^{2}}{\pi^{2}}+\frac{\tilde{\beta}_{5}^{2} \pi^{4}}{36}\right)<\left(1-\frac{\tilde{\beta}_{1}}{\pi^{\frac{3}{2}}}\right)^{2} .
$$




\section{Conclusion}

In this paper, we have studied the existence, uniqueness and exponential stability in the $p$ th moment of mild solution of impulsive neutral stochastic integro-differential equations driven by fractional Brownian motion and standard Brownian motion. Firstly, we have got sufficient conditions ensuring the existence and uniqueness of a mild solution of system (1) by using the Banach fixed point theorem. Secondly, based on the impulsive integral inequality, we have investigated the exponential stability in the $p$ th moment of mild solution of system (1), the results obtained in this paper can be generalized to a class of impulsive stochastic neutral systems, for instance, our results generalized and improved the results due to Zhou et al. [9, 24], Zhang and Ruan [14], Ma et al. [23] and Ren et al. [26].

In our future work, we mainly consider the following three problems. Firstly, we will consider dynamical systems that depend not only on past and present states, but also on derivative with delays and distributed delays, just like the case of Remark 4.6. Secondly, we will study another kind of stability of system (1), such as stochastic asymptotic stability, almost sure stability and probabilistic stability. Thirdly, inspired on our stability results of integer order impulsive NSFDEs in this paper, we will study the stability of impulsive NSFDEs in which the derivative is a fractional derivative.

\section{Acknowledgements}

Not applicable.

Funding

This work is supported by Guangxi Natural Science Foundation Project (No. 2017GXNSFBA198179), Basic Ability Promotion Project for Young and Middle-aged Teachers in Universities of Guangxi (No. 2018KY0214) and Guangxi Science and Technology Base and Specialized Talents (No. Guike AD18281026).

Availability of data and materials

Not applicable.

Competing interests

The authors declare that they have no competing interests.

\section{Authors' contributions}

All authors contributed to each part of this work equally and read approved the final manuscript.

\section{Author details}

${ }^{1}$ School of Mathematics and Computing Science, Guilin University of Electronic Technology, Guilin, China. ${ }^{2}$ College of Mathematical Sciences, University of Electronic Science and Technology of China, Chengdu, China.

\section{Publisher's Note}

Springer Nature remains neutral with regard to jurisdictional claims in published maps and institutional affiliations.

Received: 18 April 2019 Accepted: 9 October 2019 Published online: 16 October 2019

\section{References}

1. Wan, L., Duan, J.: Exponential stability of non-autonomous stochastic partial differential equations with finite memory. Stat. Probab. Lett. 78, 490-498 (2008)

2. Chen, H.: Asymptotic behavior of stochastic two-dimensional Navier-Stokes equations with delays. Proc. Indian Acad. Sci. Math. Sci. 122, 283-295 (2012)

3. Jiang, J., Shen, Y.: A note on the existence and uniqueness of mild solutions to neutral stochastic partial functional differential equations with non-Lipschitz coefficients. Comput. Math. Appl. 61, 1590-1594 (2011)

4. Boufoussi, B., Hajji, S.: Functional differential equations driven by a fractional Brownian motion. Comput. Math. Appl. 62, 746-754 (2011)

5. Ren, Y., Cheng, X., Sakthivel, R.: On time-dependent stochastic evolution equations driven by fractional Brownian motion in a Hilbert space with finite delay. Math. Methods Appl. Sci. 37, 2177-2184 (2014)

6. Luo, Q., Mao, X., Shen, Y.: New criteria on exponential stability of neutral stochastic differential delay equations. Syst. Control Lett. 55, 826-834 (2006)

7. Duc, L.H., María, J.G., Neuenkirch, A.: Exponential stability of stochastic evolution equations driven by small fractional Brownian motion with Hurst parameter in $(1 / 2,1)$. J. Differ. Equ. (2017)

8. Ren, J., Zhu, H., Zhong, S., Zhou, X.: Robust stability of uncertain Markovian jump neural networks with mode-dependent time-varying delays and nonlinear perturbations. Adv. Differ. Equ. 2016, 327 (2016) 
9. Zhou, X., Liu, X., Zhong, S.: Stochastic Volterra integro-differential equations driven by a fractional Brownian motion with delayed impulses. Filomat 31, 5965-5978 (2017)

10. Park, J.H., Shen, H., Chang, X.H., Lee, T.H.: Recent Advances in Control and Filtering of Dynamic Systems with Constrained Signals. Springer, Cham (2018). https://doi.org/10.1007/978-3-319-96202-3

11. Hale, J.K., Meyer, K.R.: A class of functional equations of neutral type. Mem. Am. Math. Soc. 76, 1-65 (1967)

12. Chen, $\mathrm{H}$.: Integral inequality and exponential stability for neutral stochastic partial differential equations with delays. J. Inequal. Appl. 2009, 297478 (2009)

13. Boufoussi, B., Hajji, S.: Neutral stochastic functional differential equations driven by a fractional Brownian motion in a Hilbert space. Stat. Probab. Lett. 82, 1549-1558 (2012)

14. Zhang, X., Ruan, D.: Exponential stability for neutral stochastic functional partial differential equations driven by Brownian motion and fractional Brownian motion. J. Inequal. Appl. 2018(1), 201 (2018)

15. Lakshmikantham, V., Bainov, D.D., Simeonov, P.S.: Theory of Impulsive Differential Equations. World Scientific, Singapore (1989)

16. Samoilenko, A.M., Perestyuk, N.A.: Impulsive Differential Equations. World Scientific, Singapore (1995)

17. Hamdy, M.A.: Approximate controllability of impulsive neutral stochastic differential equations with fractional Brownian motion in a Hilbert space. Adv. Differ. Equ. 2014, 113 (2014)

18. Arthi, G., Park, J.H.: On controllability of second-order impulsive neutral integrodifferential systems with infinite delay. IMA J. Math. Control Inf. 32, 639-657 (2015)

19. Li, Z:: Global attractiveness and quasi-invariant sets of impulsive neutral stochastic functional differential equations driven by fBm. Neurocomputing 177, 620-627 (2015)

20. Boudaoui, A., Caraballo, T., Ouahab, A.: Existence of mild solutions to stochastic delay evolution equations with a fractional Brownian motion and impulses. Stoch. Anal. Appl. 33, 244-258 (2015)

21. Li, D., Chen, G.: Exponential stability of a class of impulsive stochastic delay partial differential equations driven by a fractional Brownian motion. Int. J. Control. Autom. Syst. 15, 1561-1568 (2017)

22. Deng, S., Shu, X.B., Mao, J.: Existence and exponential stability for impulsive neutral stochastic functional differential equations driven by fBm with noncompact semigroup via Mönch fixed point. J. Math. Anal. Appl. 467, 398-420 (2018)

23. Ma, Y.K., Arthi, G., Anthoni, S.M.: Exponential stability behavior of neutral stochastic integrodifferential equations with fractional Brownian motion and impulsive effects. Adv. Differ. Equ. 2018, 110 (2018)

24. Zhou, X., Liu, X., Zhong, S.: Stability of delayed impulsive stochastic differential equations driven by a fractional Brown motion with time-varying delay. Adv. Differ. Equ. 2016, 328 (2016)

25. Arthi, G., Park, J.H., Jung, H.Y.: Existence and exponential stability for neutral stochastic integrodifferential equations with impulses driven by a fractional Brownian motion. Commun. Nonlinear Sci. Numer. Simul. 32, 145-157 (2016)

26. Ren, Y., Cheng, X., Sakthivel, R.: Impulsive neutral stochastic functional integro-differential equations with infinite delay driven by fBm. Appl. Math. Comput. 247, 205-212 (2014)

27. Kolmogorov, A.N.: The Wiener spiral and some other interesting curves in Hilbert space. Dokl. Akad. Nauk SSSR 26 $115-118(1940)$

28. Peters, E.E.: Fractal Market Analysis. Wiley, New York (1994)

29. Cheridito, P.: Mixed fractional Brownian motion. Bernoulli 7, 913-934 (2001)

30. Buckdahn, K., Jing, S.: Peng's maximum principle for a stochastic control problem driven by a fractional and a standard Brownian motion. Sci. China Math. 57, 2025-2042 (2014)

31. Guerra, J., Nualart, D.: Stochastic differential equations driven by fractional Brownian motion and standard Brownian motion. Stoch. Anal. Appl. 26, 1053-1075 (2008)

32. Mishura, Y.S., Posashkova, S.V.: Stochastic differential equations driven by a Wiener process and fractional Brownian motion: convergence in Besov space with respect to a parameter. Comput. Math. Appl. 62, 1166-1180 (2011)

33. Shevchenko, G., Shalaiko, T.: Malliavin regularity of solutions to mixed stochastic differential equations. Stat. Probab. Lett. 83, 2638-2646 (2013)

34. Da Prato, G., Zabczyk, J.: Stochastic Equations in Infinite Dimensions. Cambridge University Press, Cambridge (1992)

35. Mémin, J., Mishura, Y., Valkeila, E.: Inequalities for the moments of Wiener integrals with respect to a fractional Brownian motion. Stat. Probab. Lett. 51, 197-206 (2001)

36. Caraballo, T., Garrido-Atienza, M.J., Taniguchi, T.: The existence and exponential behavior of solutions to stochastic delay evolution equations with a fractional Brownian motion. Nonlinear Anal. 74, 3671-3684 (2011)

37. Pazy, A.: Semigroups of Linear Operators and Applications to Partial Differential Equations. Applied Mathematical Sciences, vol. 44 (1983) Springer, New York

38. Yang, H., Jiang, F.: Exponential stability of mild solutions to impulsive stochastic neutral partial differential equations with memory. Adv. Differ. Equ. 2013, 148 (2013) 